\title{
Quantitative acetylene measurements in laminar and turbulent flames using 1D Raman/Rayleigh scattering
}

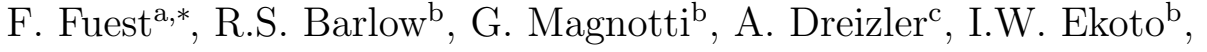 \\ J.A. Sutton ${ }^{\mathrm{a}}$ \\ ${ }^{a}$ Department of Mechanical and Aerospace Engineering, The Ohio State University, 201 \\ W. 19th Avenue, Columbus, OH, USA \\ ${ }^{b}$ Sandia National Laboratories, 7011 East Avenue, Livermore, CA, USA \\ ${ }^{c} F G$ Reactive Flows and Diagnostics, Center of Smart Interfaces, TU Darmstadt, \\ Petersenstr. 32, 64287 Darmstadt, Germany
}

\begin{abstract}
This work presents the extension of the established detection of seven major species in 1D Raman/Rayleigh measurements to incorporate acetylene $\left(\mathrm{C}_{2} \mathrm{H}_{2}\right)$ as an eighth species. Acetylene is an important soot precursor which is generated in hydrocarbon flames as an intermediate species following decomposition. It occurs over a broad temperature range in flames and has been identified as a potentially detectable species in 1D Raman/Rayleigh measurements. In this paper, we discuss the Raman spectral signature of acetylene, its temperature dependence, calibration procedures, and the interference with other Raman-active species, $\mathrm{C}_{2}$ and broadband interferences, all of which are essential to quantify for accurate data processing. In this regard, Raman measurements in laminar and turbulent dimethyl ether flames were acquired. The data are spectrally analyzed and low-temperature calibrations of the Raman response are combined with an extrapolation model
\end{abstract}

\footnotetext{
${ }^{*}$ Corresponding author

Email address: fuest.1@osu.edu (F. Fuest)
} 
for high temperatures in order to place the acetylene signals on an absolute scale. Single-shot and conditional-mean values are presented as a function of mixture fraction for a laminar partially-premixed DME/air flame. Measurements from the laminar flame are compared to a 1D laminar flame calculation to assess the accuracy of the calibration and analysis procedures. Finally, detection limits, signal-to-noise, and signal-to-interference ratio of the $\mathrm{C}_{2} \mathrm{H}_{2}$ measurement are discussed, and single-shot measurements of acetylene in a turbulent DME/air jet flame are demonstrated.

Keywords: Raman spectroscopy, acetylene, dimethyl ether, combustion diagnostics

\section{Introduction}

In the past decades, 1D Raman/Rayleigh scattering has become a standard tool to determine the thermo-chemical state in turbulent flames [1]. For simple fuels such as hydrogen or methane, up to seven major species $\left(\mathrm{CO}_{2}, \mathrm{O}_{2}, \mathrm{CO}, \mathrm{N}_{2}, \mathrm{CH}_{4}, \mathrm{H}_{2} \mathrm{O}, \mathrm{H}_{2}\right)$, temperature, and the corresponding gradients have been measured simultaneously and instantaneously, providing valuable information about turbulence-chemistry interaction $[2,3]$. Such measurements have been combined with independent velocity measurements in various turbulent flames to create extensive data sets that have been used for assessment and validation of turbulent combustion models $[4,5]$. In recent years, collaborative efforts have been made to extend Raman/Rayleigh scattering methods beyond hydrogen and methane fuels to larger and more complex molecules. For this purpose, dimethyl ether (DME) has been selected as a promising fuel candidate, primarily due to its relatively low soot levels 
as compared to other gaseous hydrocarbon fuels, such as ethylene or ethane, and its potential as a realistic transportation fuel in compression-ignition engines. Initial results of measurements in laminar and turbulent DME flames have been presented previously [6-8]. Most recently, preliminary 1D Raman/Rayleigh results from a new validation data set of partially-premixed DME flames, introduced by Frank et al. [9], have been presented [10].

Since higher levels of intermediate hydrocarbons are produced in all higher hydrocarbon-fueled flames as compared to methane flames, additional information beyond the currently-measured seven major species becomes important. As outlined in [6, 7], unknown intermediate hydrocarbon concentrations can have significant impact on uncertainties in measurements of species mass fractions, mixture fraction, and temperature in Raman and Rayleigh experiments. In order to reduce these uncertainties, acetylene $\left(\mathrm{C}_{2} \mathrm{H}_{2}\right)$ was determined to be one viable candidate for additional measurement [6]. Measured acetylene concentrations also would be beneficial to investigate additional chemistry, such as the formation of polycyclic aromatic hydrocarbons and soot in turbulent flames, as done previously in laminar flames [11-16]. To date, all previous studies have presented time- and/or spatially-averaged results in order to allow for reasonable signal-to-noise ratios. Consequently, no instantaneous and spatially-resolved measurements in turbulent flames have been reported. As one example of previously-reported results, Mokhov et al. $[13,14]$ applied spontaneous Raman scattering to detect acetylene in a laminar methane/air flame. For the reported point measurements they used a $1 \mathrm{kHz}, 25 \mathrm{~s}$ exposure time and 20 image-accumulation yielding a reported signal-to-noise ratio of 5 . 
In this work we present instantaneous concentration measurements of acetylene in laminar and turbulent flames with spatial resolution high enough to resolve concentration gradients in turbulent flames. Modifications to existing 1D spontaneous Raman/Rayleigh scattering experimental procedures and analysis, including calibration methods, are developed to allow the inclusion of $\mathrm{C}_{2} \mathrm{H}_{2}$ in addition to the established instantaneous measurements of temperature and seven major species. Furthermore, we present the corresponding signal-to-noise and error analysis for the single-shot detection of acetylene. The experimental setup is described in Section 2. Section 3 describes relevant spectroscopic considerations to interpret the Raman species measurement in DME/air flames with a particular focus on acetylene. The spectral position and possible signal interferences from other sources are discussed. In order to provide accurate measurements at different temperatures, changes in the integrated Raman and interference signals with temperature must be considered. The corresponding temperature corrections for acetylene and interferences are presented in Section 4 and 5. Signal-to-noise and signal-to-interference ratios and possible systematic errors are discussed in Section 6. Results in laminar and turbulent flames are presented in Section 7 and conclusions in Section 8.

\section{Experimental method}

The measurements presented in this paper were performed at the Combustion Research Facility at Sandia National Laboratories. The most pertinent details of the experimental setup and procedures are given below; for a 
detailed description see [6, 17-19]. The 532-nm output of four sequentiallyfired, frequency-doubled Nd:YAG lasers were combined and used to determine major species mole fractions and temperature along a 6.16-mm line segment via spontaneous Raman/Rayleigh scattering. In order to avoid optical breakdown at the probe volume, reduced laser power and a three-cavity optical pulse stretcher [20] with 38\% partial reflectors and delays of approximately 36, 18, and $9 \mathrm{~ns}$ were used. This resulted in a pulse width for each laser of $83 \mathrm{~ns}$ (FWHM) and an overall duration of $0.5 \mu \mathrm{s}$. A combined energy of $1 \mathrm{~J} /$ pulse is focused by a $500-\mathrm{mm}$ lens to a beam waist of $\sim 200 \mu \mathrm{m}$ as determined from the $1 / \mathrm{e}^{2}$ thickness of the 1D Rayleigh scattering image. Single-shot precision in the Rayleigh temperature measurement varies between $0.25 \%$ and $1 \%$ for room and flame temperatures, respectively.

The emitted Raman- and Rayleigh-scattered light is collected at $90^{\circ}$ with respect to the incident laser pulses with a combination of $\mathrm{f} / 2$ and $\mathrm{f} / 4$ achromats and focused into a custom spectrometer as described in [21]. Within the spectrometer the Raman and Rayleigh signals were separated using a dichroic beam splitter. The reflected light was collected on the Rayleigh CCD camera and the transmitted signal was sent through a thin-film polarizer and dispersed via a high-throughput transmission grating (1200 lines/mm). A lownoise cryogenically-cooled CCD camera (Roper Scientific, VersArray 1300) in conjunction with custom-built rotating wheel shutters, were used to collect the dispersed Raman signals from 550-700 nm with exposure times as short as $3.9 \mu \mathrm{s}$ (FWHM). The data were acquired both spectrally resolved $\left(\sim 3 \mathrm{~cm}^{-1} /\right.$ pixel) and using additional spectral on-chip hardware binning to decrease the camera readout noise. In the spatial direction ten-pixel on-chip 
hardware binning was applied, yielding 60 spatial Raman superpixels along the line segment with a spatial resolution of $102.6 \mu \mathrm{m} /$ pixel. The images were processed using the hybrid matrix inversion method with extensions for processing DME data as outlined in $[6,7,18]$.

Air, cold gases diluted with nitrogen, and laminar methane/air flat flames were used for calibration of major species $\left(\mathrm{CO}_{2}, \mathrm{O}_{2}, \mathrm{CO}, \mathrm{N}_{2}, \mathrm{DME}, \mathrm{H}_{2} \mathrm{O}\right.$, $\mathrm{H}_{2}$ ) signals and temperature. The integrated Raman response of acetylene was determined from previous measurements [22], where the Raman signal from acetylene was measured from room temperature to $700 \mathrm{~K}$, using a silicon carbide heater within a quartz tube as illustrated in Fig. 1.

A mixture of 1:5 $\mathrm{C}_{2} \mathrm{H}_{2}$ in nitrogen (by volume) was used for the heatedflow measurements. Averaged spectra were acquired from the probe volume centered just above the heater outlet. Temperature in the heated $\mathrm{C}_{2} \mathrm{H}_{2}$ flow was determined from the ratio of the Rayleigh signal from a roomtemperature flow at the same mixture composition to that from the heated flow. Laminar and turbulent partially-premixed jet flames of 20\% DME (onchip binning) and 28\% DME (spectrally resolved) mixed with air were used for the investigation of quantitative acetylene concentration measurements in flames and the feasibility of quantitative single-shot measurements of acetylene in turbulent DME flames. Partially-premixed fuel and air issued from 7.72-mm and 7.45-mm-diameter circular tubes, where the Reynolds numbers based on tube diameter were 1,530 and 29,300 for the laminar and turbulent flames, respectively. 


\section{Raman scattering of acetylene}

To extend the current detection of Raman signals from seven major species to include acetylene, spectral regions corresponding to $\mathrm{C}_{2} \mathrm{H}_{2}$ in the recorded Raman shift range of $750-4600 \mathrm{~cm}^{-1}$ were identified as reported previously $[6,7]$. Two fundamental vibration modes occur in the considered spectral range [23]. The $\mathrm{C} \equiv \mathrm{C}$ vibrational Raman shift of $1973.8 \mathrm{~cm}^{-1}$ is po-

sitioned between the current channels of $\mathrm{O}_{2}$ and CO. The Raman band of the $\mathrm{C}-\mathrm{H}$ vibration at $3373.7 \mathrm{~cm}^{-1}$ is located close to the $\mathrm{H}_{2} \mathrm{O}$ channel and is about nine times weaker than the $\mathrm{C} \equiv \mathrm{C}$ vibration $[24,25]$.

Figure 2 shows an averaged Raman spectrum at $1290 \mathrm{~K}$ acquired from a rich laminar DME/air jet flame [6, 7]. All Raman channels are indicated by vertical dash-dotted lines. The signal within these channels is integrated before processing the data, either by software-binning or during the data acquisition by on-chip hardware binning. A new channel has been introduced to detect the signal of the $\mathrm{C} \equiv \mathrm{C}$ vibration and is marked red in Fig. 2. Also, corresponding species are shown in percent volume to allow for comparison of relative signal intensities which are proportional to their volume fractions. According to laminar flame calculations at these conditions [6, 7], the acetylene signal peaking at $1950 \mathrm{~cm}^{-1}$ corresponds to a mole fraction of 0.006 (or a volume percentage of $0.6 \%$ ).

The spectrally-integrated signal ratio of $12 \% \mathrm{CO}$ and $0.6 \% \mathrm{C}_{2} \mathrm{H}_{2}$ in Fig. 2 is approximately three. Accordingly, this band of $\mathrm{C}_{2} \mathrm{H}_{2}$ has a Raman cross section that is approximately six to seven times larger than that of CO. This large Raman cross section allows for smaller detection limits compared to major intermediate species such as $\mathrm{CO}$ or $\mathrm{H}_{2}$ as discussed below. 
Important for data-processing is the observation that the $\mathrm{C}_{2} \mathrm{H}_{2}$ feature is well separated from other channels and therefore does not interfere with other Raman signals. Possible signals from $\mathrm{CO}$ or $\mathrm{H}_{2}$ on the $\mathrm{C}_{2} \mathrm{H}_{2}$ channel also are found to be negligible, based on theoretical calculations of the Raman spectra of $\mathrm{CO}$ and $\mathrm{H}_{2}$ convolved with the transfer function for the present system. However, a strong crosstalk assigned to $\mathrm{C}_{2}$ and broadband interference from polycyclic aromatic hydrocarbon (PAH) molecules as well as a small residual background most likely from flame luminosity needs to be accounted for in the data processing. The $\mathrm{C}_{2}$ and PAH crosstalk impacts all Raman channels and is generally found in rich hydrocarbon flames, e.g. [26]. The interfering signal begins to be important on the fuel-rich side of the flame at temperatures between $1000 \mathrm{~K}$ and $1200 \mathrm{~K}$, peaks between 1600-1800 K, then decreases significantly or even disappears before reaching the point of maximum temperature in the flame. The strongest features of the interfering signal are observed around $900 \mathrm{~cm}^{-1}$ which are assigned to $\mathrm{C}_{2}$-band emissions from the $(3,2),(2,1)$, and $(1,0)$ vibrational transitions at $741 \mathrm{~cm}^{-1}, 883 \mathrm{~cm}^{-1}$, and $1042 \mathrm{~cm}^{-1}$, respectively. They appear on the leftmost channel in the spectrum shown in Fig. 2 which is marked as 'F560' (fluorescence around $560 \mathrm{~nm}$ ) and are mixed with a feature of DME around $900 \mathrm{~cm}^{-1}$ and pure rotational Raman transitions from $\mathrm{H}_{2}[6,7]$. Weaker $\mathrm{C}_{2}$ emissions resulting from $\Delta v=2$ and $\Delta v=3$ transitions affect the majority of the Raman spectrum. At a temperature of $1290 \mathrm{~K}$ a small level of this interference underlies the spectrum shown in Fig. 2, i.e. 15-20\% of the signal within the $\mathrm{C}_{2} \mathrm{H}_{2}$ channel is attributed to the $\mathrm{C}_{2}$ and broadband interference which must be removed during data processing. This crosstalk correction is 
based on the integrated signal measured at F560 and is strongly temperature dependent for most species $[6,7]$. In practice, the temperature dependence needs to be determined experimentally, which can be very time-consuming and potentially unfeasible for very high interferences associated with flames using ethylene or ethane fuels, where the interference can be orders of magnitude higher than that in methane flames. However, the DME/air flames considered in this work have only 1.5 to 2 times higher interferences than similar $\mathrm{CH}_{4}$ /air flames and thus it was possible to transfer established meth-

ods $[6,7]$ to correct the acetylene channel for the F560 interferences and residual background luminosity as shown below.

\section{Raman signal calibration and temperature dependence}

For accurate data-processing using the iterative matrix inversion method, temperature-dependent polynomials or 'lookup' tables are needed to describe acetylene and crosstalk signal variations with temperature. These are determined for six of seven major species by quantum mechanical calculations [6, 18]. Currently, no reliable calculations for hydrocarbons are available and they are treated with experimental calibration $[6,7]$.

A simple approach to estimate the temperature dependence of the acetylene signal is to assume that the two hydrogen atoms in $\mathrm{C}_{2} \mathrm{H}_{2}$ are negligible perturbations and the $\mathrm{C}_{2}$ triple-bond may be treated using Placzek's double harmonic approximation for the molecule's potential function and polariz-

ability [27]. Also, rotational energy levels and transitions are neglected and the temperature dependence of the Raman cross section is described by Eq. 
(5.23) in $[28]$,

$$
\left(\frac{\partial \sigma}{\partial \Omega}\right)=\frac{h\left(\nu_{0}-\nu_{k}\right)^{4}\left\{\left(a^{\prime}\right)^{2}+\frac{4}{45}\left(\gamma^{\prime}\right)^{2}\right\}}{8 m c^{4} \nu_{k}\left[1-\mathrm{e}^{-h c \omega_{\mathrm{e}} / k T}\right]},
$$

where we omitted the index $z z$ that denotes the illumination-observation geometry, $\omega_{e}$ is the bond-specific equilibrium frequency, $h$ is the Planck constant, $m$ is the mass of the molecule, $c$ is the speed of light, $k$ is the Boltzmann constant, $\nu_{0}$ is the frequency of the laser, and $\nu_{k}$ is the frequency of the energy difference of the vibrational transition, i.e. $\nu_{k}=\omega_{e} c$. Since the harmonic approximation neglects transition-specific energy differences, the Raman spectrum is reduced to a single line; thus, the relative temperature dependence of the total Raman cross section of the Q-branch is given by

$$
\left(\frac{\partial \sigma}{\partial \Omega}\right) /\left(\frac{\partial \sigma}{\partial \Omega}\right)_{T_{0}}=\frac{1-\mathrm{e}^{-h c \omega_{\mathrm{e}} / k T_{0}}}{1-\mathrm{e}^{-h c \omega_{\mathrm{e}} / k T}},
$$

where $T_{0}$ is an arbitrary reference temperature. For a single line the detected Q-branch Raman signal $S_{\text {Ram,Q }}$ is proportional to the Raman cross section and Eq. 2 is written as

$$
S_{\text {Ram }, \mathrm{Q}}(T)=S_{\text {Ram }, \mathrm{Q}}\left(T_{0}\right) \frac{1-\mathrm{e}^{-h c \omega_{\mathrm{e}} / k T_{0}}}{1-\mathrm{e}^{-h c \omega_{\mathrm{e}} / k T}} .
$$

It can be shown for simple molecules (e.g. for $\mathrm{N}_{2}$ and $\mathrm{CO}$ ) that Eq. (3) is a close approximation to much more sophisticated calculation methods assuming that the majority of the Raman-scattered signal is collected. This is demonstrated in Fig. 3 for $\mathrm{N}_{2}$ and $\mathrm{CO}$ where comparisons are shown between the harmonic oscillator approximation and detailed quantum mechanical calculations of the Raman response as outlined in [18]. In both cases, the detailed calculations show a local minimum of integrated intensity near 
$600 \mathrm{~K}$, whereas the harmonic oscillator approximation does not. In addition, the harmonic-oscillator model overestimates the temperature dependence by a few percent at high temperatures.

As reported in [18] measurements of the temperature dependence of the Raman response of $\mathrm{N}_{2}$ and $\mathrm{CO}$ are in very good agreement with the detailed calculations. The measured temperature dependence of the Raman response for acetylene shows a trend between $290 \mathrm{~K}$ and $700 \mathrm{~K}$ similar to those of $\mathrm{N}_{2}$ and $\mathrm{CO}$; that is, a local minimum of integrated intensity near $600 \mathrm{~K}$. Figure 4 compares the measured temperature dependence with an estimated Raman response using a simple harmonic oscillator model for a frequency of $1973.8 \mathrm{~cm}^{-1}$ and a polynomial curve that fits the measurements at low temperature and extrapolates to high temperature in a manner similar to the detailed calculations for $\mathrm{N}_{2}$ and $\mathrm{CO}$ (solid line). It is noted that the polynomial extrapolation from the measured data remains within six percent of the simple harmonic oscillator for temperatures up to $2000 \mathrm{~K}$. Two additional polynomial curves are shown in Fig. 4, denoted 'Polynomial low' and 'Polynomial high'. These polynomials are used as extreme cases for the sensitivity analysis as discussed in Sec. 7. The measurements at $290 \mathrm{~K}$ yielded an integrated signal intensity ratio of $S_{\mathrm{C}_{2} \mathrm{H}_{2}} / S_{\mathrm{N}_{2}}=5.6$, as compared to $S_{\mathrm{C}_{2} \mathrm{H}_{2}} / S_{\mathrm{N}_{2}}=6$ measured previously by Stephenson [24] for relative peak intensities between acetylene and the Q-branch signal from nitrogen at similar temperature conditions. The relatively high Raman cross section of acetylene is beneficial because concentrations are low compared to those typically accessed by Raman scattering.

Since acetylene is very prone to produce optical breakdown in the probe 
volume only $110 \mathrm{~mJ} /$ pulse were used for the inline heater measurements as compared to $1 \mathrm{~J} /$ pulse for the flame measurements. In order to account for the resulting difference in signal, the calibration factor from acetylene was scaled according to the ratio of the Raman response from nitrogen in both measurements. The constructed curve 'Polynomial' in Fig. 4 was used for the temperature dependence in generating the results presented below.

\section{Treatment of interferences}

At the location of peak interference contributions of $70 \% \pm 10 \%$ were measured in the laminar flame by rotating the thin-film polarizer within the spectrometer by 90 degrees such that the vertically-polarized Raman signal was rejected and the horizontally-polarized signal from depolarized interference was measured. Contributions from the horizontally-polarized Raman signal were small (less than 3\%). The actual adjustment of the F560 crosstalk factor was applied in mixture fraction space such that it stayed within the limits of $70 \% \pm 10 \%$ and minimized the distortion to the acetylene profile.

The temperature dependence of the $\mathrm{C}_{2}$ and broadband interference correction was assumed to be the same as that derived for $\mathrm{CO}[6]$ previously and is shown in Fig. 5 as the solid black line. The $\mathrm{C}_{2} \mathrm{H}_{2} \leftarrow \mathrm{F} 560$ curve in Fig. 5 displays a range of signal values from crosstalk relative to $290 \mathrm{~K}$ varying between 4.5-12 in the relevant temperature region of $1000 \mathrm{~K}$ to $1800 \mathrm{~K}$. As discussed below the peak values for $\mathrm{C}_{2} \mathrm{H}_{2}$ were measured in the experiment for temperatures between $1500 \mathrm{~K}$ to $1600 \mathrm{~K}$, which were used as the calibration-temperature range for the $\mathrm{C}_{2} \mathrm{H}_{2} \leftarrow \mathrm{F} 560$ crosstalk correction.

Systematic corrections for variations of the temperature dependencies 
along the Raman line segment were applied for the $\mathrm{C}_{2} \mathrm{H}_{2} \leftarrow \mathrm{F} 560$ interference correction due to an effective wavenumber shift induced by an optical bowing effect [18]. A linear correction of up to 1.25 was applied to correct for the spatial variation in wavenumber shift. Finally, the Raman signal on the water channel was used to correct for a small remaining background on the acetylene channel, which most likely corresponds to residual flame luminosity. It is noted that this correction is not a classical crosstalk from Raman scattering although the Raman signal from water is used. This is indicated by ' $\left(\mathrm{H}_{2} \mathrm{O}\right)^{\prime}$ instead of ' $\mathrm{H}_{2} \mathrm{O}$ ' in the crosstalk-symbol $\mathrm{C}_{2} \mathrm{H}_{2} \leftarrow\left(\mathrm{H}_{2} \mathrm{O}\right)$. The temperature dependence for this correction is shown in Fig. 5 as the solid red line.

\section{Signal-to-noise and error analysis}

Following on previous work, e.g. $[6,7,18]$, the matrix inversion method was extended to include an additional $\mathrm{C}_{2} \mathrm{H}_{2}$ channel and the necessary signal crosstalks terms in the data-processing. Calibration procedures and signal response characteristics as outlined in Sections 4 and 5 were applied to hardware-binned data taken in the laminar DME/air jet (20\% DME) at an axial position $10 \mathrm{~mm}$ downstream of the nozzle exit and at radial positions corresponding to $r=0$ to $12 \mathrm{~mm}$. The new binning region was included to the former binning scheme of the CCD camera, as shown in Fig. 2.

Figure 6a shows the mean acetylene mole fractions measured by Raman scattering and the mean temperature profile measured by Rayleigh scattering as a function of radial position on the rich side of the flame. The error bars on the acetylene measurements represent one standard deviation from 150 
laser shots. Each Raman pixel corresponds to $0.103 \mathrm{~mm}$ in physical space. In addition, results from data-processing without applying the F560 interference correction are shown. Figure $6 \mathrm{~b}$ shows the corresponding signal-to-noise ratios derived from the mean values divided by the standard deviation of the 150 single-shot measurements. Dash-dotted, vertical lines mark the detection limit defined as $\mathrm{SNR} \geq 5$, which is in consideration of the application to singleshot measurements in turbulent flames. Figures $6 \mathrm{a}$ and $6 \mathrm{~b}$ demonstrate that acetylene can be measured using single-shot detection within a wide range of concentrations and temperatures in this flame with SNR values up to 9. Under rich conditions, the detection limit of acetylene is as low as $0.3 \%$ at $840 \mathrm{~K}$ and $0.64 \%$ at $1770 \mathrm{~K}$ with a peak measured value corresponding to approximately $0.9 \%$ at $1520 \mathrm{~K}$.

Figure $6 \mathrm{c}$ shows the percentage of the interference of the total measured signal on the acetylene channel arising from the background ('bgr'), fluorescence and broad band crosstalk ('F560'), and residual interferences, e.g. from flame luminosity which are determined from the water channel (' $\left.\left(\mathrm{H}_{2} \mathrm{O}\right)^{\prime}\right)$. The results in Fig. 6c show that significant contributions from all three interference corrections are subtracted from the total signal measured on the acetylene channel. First, around $r=2 \mathrm{~mm}$, the entire signal is attributed to background signal which is measured within the 'bgr' channel indicated in Fig. 2. Once acetylene starts to form around $r=2.5 \mathrm{~mm}$, the Raman scattering signal from acetylene accounts for the majority of the total measured signal and the added contributions from all three interferences become as small as $20 \%$ for radial positions corresponding to $r=3$ to $3.5 \mathrm{~mm}$. While the 'bgr' contribution stays approximately constant at $10 \%$ until $r=4.5 \mathrm{~mm}, \mathrm{C}_{2}$ 
interferences (F560) are continuously increasing with increasing $\mathrm{C}_{2} \mathrm{H}_{2}$ mole fraction to approximately $60 \%$ of the collected signal. The portion of the signal originating from the Raman scattering of acetylene does not increase beyond $40 \%$ for increasing radial positions. At very low acetylene mole fractions near the peak temperature $(\mathrm{r}>5 \mathrm{~mm})$ the residual signal and background luminosity corrections are both as high as $50 \%$ of the total collected signal. It is seen in Fig. 6b that the F560 correction does not affect the detection limit of $\mathrm{C}_{2} \mathrm{H}_{2}$ at the lower temperatures near the centerline of the flame. However, the application of the F560 correction acts to reduce the SNR values with increasing radial position (and increasing temperatures) due to the subtraction of a significant percentage of the total measured signal. Furthermore, it is noted that without the F560 correction, there is a significant difference in the shape of the acetylene mole fraction profiles as shown in Fig. 6a. The results without the F560 correction display a larger temperature dependence than the results using the F560 correction. The gradients of the acetylene profile are steeper on both sides, the full width at half maximum is decreased by approximately a factor of two. The results in Figs. 6a-c emphasize the importance of the $\mathrm{F} 560\left(\mathrm{C}_{2}\right.$ and broadband fluorescence) correction.

The main sources of uncertainty in the derived $\mathrm{C}_{2} \mathrm{H}_{2}$ mole fractions are from the extrapolated temperature dependence of the Raman response (constructed curve in Fig. 4) and the interference corrections. At a temperature of $1200 \mathrm{~K}$ on the fuel-rich side of the flame, the corrections for fluorescence interference and background luminosity are both small and the uncertainty in the derived $\mathrm{C}_{2} \mathrm{H}_{2}$ mole fraction is estimated to be $15 \%$. The peak $\mathrm{C}_{2} \mathrm{H}_{2}$ mole fraction is strongly affected by fluorescence, and uncertainty in the peak 
mole fraction is estimated to be $25 \%$.

\section{Results}

Experimental results for seven major species, acetylene, and temperature from a laminar DME/air jet flame (20\% DME) are shown in Figs. 7 and 8. Figure 7 shows the mean mole fraction values from 150 single-shot measurements as a function of radial position, while Fig. 8 shows the conditional average in mixture fraction space. The corresponding experimental results for acetylene are shown in Figs. 9 and 10. To avoid ambiguities in mixture fraction values close to $\xi=1$, results for $\xi>0.85$ were conditioned on reaction progress, determined as $c=\left(T-T_{\text {fuel }}\right) /\left(T_{\text {adiabatic }}-T_{\text {fuel }}\right)$, instead of mixture fraction. For consistency, this approach was used for all conditionally-averaged species and temperature profiles shown in Figs. 8 and 10. The experimental data are compared to a 1D laminar opposed jet flame calculation from CHEMKIN [29] using multi-component transport and the DME reaction mechanism by Zhao et al. [30]. To compare the experimental results of the coaxial jet flame with 1D opposed-flow jet calculations (i.e., Figs. 7 and 8), the flow velocities in the calculation were adjusted such that the calculated temperature profile matched the measured width of the temperature profile in physical space. This process resulted in a strain rate of $28 \mathrm{~s}^{-1}$ for the calculation based on the strain rate formulation from Seshadri and Williams [31]. It is noted that there is good agreement between the experimental results and the calculations for all seven major species and temperature in both physical and mixture fraction space. While minor differences between the experimental results and calculations are found in the 
major species and temperature profiles, it is concluded that laminar 1D calculations are suitable for the prediction of temperature and major species in these flames. However, the measured peak values and mole fraction gradients of acetylene are significantly under-predicted by the calculations as compared to the results of the major species and temperature. For example, the peak acetylene mole fraction is under-predicted by approximately $50 \%$, which is outside of the estimated uncertainty of the present measurements. It should be noted that comparisons of measured and modeled acetylene profiles in low-pressure premixed DME flames by Wang et al. [32] have shown very good agreement in rich flames, but differences of a factor of two or more in near-stoichiometric and lean flames. Bennett et al. [33] found lower concentrations in $\mathrm{C}_{2} \mathrm{H}_{2}$ mass spectrometry compared to their calculations. However, it should be considered that acetylene may be more sensitive than the major species to geometric differences and transport effect in jet flames versus opposed-flow flames. These issues will require further investigation.

Figure 11 shows the sensitivity of the derived acetylene mole fractions on the Raman signal response curve of acetylene (Fig. 4) and the two crosstalk corrections based on the signals measured at the F560 and $\mathrm{H}_{2} \mathrm{O}$ Raman channels. Conditional mean values of temperature and acetylene mole fraction from the laminar flame are presented as a function of mixture fraction in conjunction with the results from the 1D laminar flame calculation.

The experimental results from Fig. 10 are shown as the gold points, while the calculations are shown as the gold solid line. Experimental results derived using the two extreme bounds of the temperature-dependent Raman response curve, denoted 'Polynomial low' and 'Polynomial high' in Fig. 4, are shown as 
the curves with the blue and cyan points, respectively. The results in Fig. 11 show that even large differences in the response curves have only small effects on the derived $\mathrm{C}_{2} \mathrm{H}_{2}$ mole fraction values. Using the 'steeper' response curve ('Polynomial high') reduces the derived acetylene mole fraction while the flat response curve ('Polynomial low') results in increased acetylene mole fractions.

To assess the sensitivity of the derived acetylene mole fraction values to the F560 background correction, new results are derived where the F560 corrections developed in Sec. 5 are artificially increased and decreased by 20\%. These results are denoted in Fig. 11 as '+20\%F560' and '-20\%F560', respectively. It is seen that this correction has a larger impact on the derived $\mathrm{C}_{2} \mathrm{H}_{2}$ mole fraction values than the temperature-dependent Raman response curve. By changing the F560 correction by +/- 20\%, e.g. the peak acetylene mole fractions change by approximately $35 \%$ and 15\%, respectively. Finally, the sensitivity of the $\mathrm{C}_{2} \mathrm{H}_{2}$ measurements to the correction of the remaining background, presumably from flame luminosity, was assessed. As discussed in Sec. 5, the Raman signal from the $\mathrm{H}_{2} \mathrm{O}$ channel within the region of $0.1<\xi<0.4$ was used to determine the correction $\mathrm{C}_{2} \mathrm{H}_{2} \leftarrow\left(\mathrm{H}_{2} \mathrm{O}\right)$. The brown points in Fig. 11 show the results, where the $\mathrm{C}_{2} \mathrm{H}_{2} \leftarrow\left(\mathrm{H}_{2} \mathrm{O}\right)$ correction is not applied. The acetylene mole fractions are not sensitive to this correction except for regions where the local $\mathrm{C}_{2} \mathrm{H}_{2}$ mole fraction is less than $20 \%$ of the peak $\mathrm{C}_{2} \mathrm{H}_{2}$ mole fraction. Without applying the $\mathrm{C}_{2} \mathrm{H}_{2} \leftarrow\left(\mathrm{H}_{2} \mathrm{O}\right)$ correction, the peak acetylene mole fraction changes by less than $5 \%$.

Finally, the methodology developed in this work was applied to 1D Raman/Rayleigh measurements in a turbulent, partially-premixed DME/air 
(20\% DME) jet flame operating at a Reynolds number of 30,750 (DMED) [10]. For the first time acetylene mole fractions were measured instantaneously in a turbulent flame. Results from 2400 single-shot measurements are shown in Fig. 12 as a scatter plot and the corresponding conditional mean values as a function of mixture fraction. Also shown are the conditional mean values of temperature. A peak conditional mean value of 0.006 mole fraction is measured which corresponds to a decrease of approximately $50 \%$ as compared to the laminar case shown in Fig. 10. As expected, the turbulent flame results demonstrate large fluctuations in $\mathrm{C}_{2} \mathrm{H}_{2}$ mole fraction. In addition, a 'triangle-like' profile, confined to $0.4<\xi<0.1$ is noted with no apparent effects of differential diffusion at rich conditions near $\xi=1$. Future work will focus on measurements of acetylene concentrations at different axial locations in laminar and turbulent flames of dimethyl ether and other hydrocarbon fuels. In addition, the simultaneous detection of acetylene may allow for the reduction of uncertainties in derived quantities from 1D Raman/Rayleigh measurements such as temperature, species mass fractions, atom ratios, and mixture fraction as described in $[6,7]$. This is an important aspect in terms of combustion model validations.

\section{Summary and conclusions}

In this work we present quantitative, single-shot measurements of acetylene mole fractions using one-dimensional spontaneous Raman/Rayleigh scattering in laminar and turbulent DME/air flames. This work represents a valuable extension of the Raman/Rayleigh methodology, which had previously

yielded measurements of seven major species and temperature. Signal-to- 
noise ratios of up to 9 were achieved using single-shot detection at $1 \mathrm{~J} /$ pulse and a spatial resolution of $\sim 100 \mu \mathrm{m}$ along a 1D line segment. Detection limits, defined as measurements with $\mathrm{SNR} \geq 5$ were determined to be 0.003 mole fraction at $840 \mathrm{~K}$ (interference-free), and 0.0064 mole fraction at $1770 \mathrm{~K}$ with large interference. The composition of the signal measured within the new acetylene channel was investigated in detail and interference contributions of up to $70 \%$ were found.

Temperature-dependent response curves for the acetylene Raman signal and for two crosstalk corrections were introduced to allow for accurate data processing within the applied matrix inversion method. In the laminar case it was possible to estimate an error of $\pm 15 \%$ in the measured acetylene values at interference-free regions and $\pm 25 \%$ for the peak acetylene value with high interferences. Although good agreement was found in the measured profiles of seven major species and temperature as compared to a laminar flame calculation, measured acetylene profiles were found to be systematically higher by as much as $50 \%$ than calculated values.

Although the main focus of this work is on the development of a methodology for single-shot detection of acetylene in turbulent flames, it is noted that spatial resolution and/or signal-to-noise characteristics of the shot-averaged measurements (applicable to laminar flames) are significantly higher than acetylene measurements previously reported in the literature.

\section{Acknowledgements}

Work at Ohio State University was supported by the Combustion Energy Frontier Research Center funded by the US department of Energy, Office of 
Science, BES under Award DE-SC0001198. Work performed at Sandia was supported by the Division of Chemical Sciences, Geosciences and Biosciences, Office of Basic Energy Sciences, US Department of Energy. Sandia National Laboratories is a multiprogram laboratory operated by Sandia Corporation, a Lockheed Martin Company, for the United States Department of Energy under contract DE-AC04-94-AL85000. A portion of the work was supported by Deutsche Forschungsgemeinschaft SFB 568 and EXC 259. The help of R. Harmon during the experiments is gratefully acknowledged.

[1] R. S. Barlow, Laser diagnostics and their interplay with computations to understand turbulent combustion, Proc. Combust. Inst. 31 (1) (2007) $49-75$.

[2] D. Geyer, A. Kempf, A. Dreizler, J. Janicka, Turbulent opposed-jet flames: A critical benchmark experiment for combustion LES, Combust. Flame 143 (4) (2005) 524-548.

[3] G. Wang, A. N. Karpetis, R. S. Barlow, Dissipation length scales in turbulent nonpremixed jet flames, Combust. Flame 148 (1-2) (2007) 62 $-75$.

[4] TNF, International Workshop on Measurement and Computation of Turbulent Nonpremixed Flames (TNF) URL http://www.sandia.gov/ TNF.

[5] D. C. Haworth, Progress in probability density function methods for turbulent reacting flows, Prog. Ener. Combust. 36 (6) (2010) 168-259. 
[6] F. Fuest, 1D Raman/Rayleigh-scattering and CO-LIF measurements in laminar and turbulent jet flames of dimethyl ether using a hybrid data reduction strategy, Ph.D. thesis, Technische Universität Darmstadt, Germany, URL http://tuprints.ulb.tu-darmstadt.de/ 2773/, 2011.

[7] F. Fuest, R. S. Barlow, J.-Y. Chen, A. Dreizler, Raman/Rayleigh scattering and CO-LIF measurements in laminar and turbulent jet flames of dimethyl ether, Combust. Flame 159 (2012) 2533-2562.

[8] K. N. Gabet, H. Shen, R. A. Patton, F. Fuest, J. A. Sutton, A comparison of turbulent dimethyl ether and methane non-premixed flame structure, Proc. Combust. Inst. 34 (1) (2012) 1447 - 1454.

[9] J. H. Frank, A. G. Hsu, J. Kuhl, Turbulent Partially Premixed Dimethyl Ether/air Jet Flames: A New Series Of Target Flames For Experiments And Modeling, Joint Meeting- US Sections of the Combustion Institute 2 (2011) 911-916.

[10] F. Fuest, G. Magnotti, R. S. Barlow, J. A. Sutton, Scalar structure of turbulent partially-premixed dimethyl ether/air jet flames, Proc. Combust. Inst. URL http://dx.doi.org/10.1016/j.proci.2014.07.062.

[11] S. Gersen, A. Mokhov, H. Levinsky, Extractive probe/TDLAS measurements of acetylene in atmospheric-pressure fuel-rich premixed methane/air flames, Combust. Flame 143 (3) (2005) 333 - 336.

[12] E. W. Kaiser, Measured and computer-simulated hydrocarbon and hydroxyl radical profiles in fuel-rich atmospheric-pressure flat flames: re- 
actions of methane and acetylene, J. Phys. Chem. 94 (11) (1990) 44934499.

[13] A. V. Mokhov, S. Gersen, H. B. Levinsky, Spontaneous Raman measurements of acetylene in atmospheric-pressure methane/air flames, Chem. Phys. Lett. 403 (4-6) (2005) $233-237$.

[14] A. Mokhov, B. Bennett, H. Levinsky, M. Smooke, Experimental and computational study of $\mathrm{C}_{2} \mathrm{H}_{2}$ and $\mathrm{CO}$ in a laminar axisymmetric methane/air diffusion flame, Proc. Combust. Inst. 31 (1) (2007) 997 $-1004$.

[15] S. Wagner, M. Klein, T. Kathrotia, U. Riedel, T. Kissel, A. Dreizler, V. Ebert, In situ TDLAS measurement of absolute acetylene concentration profiles in a non-premixed laminar counter-flow flame, Appl. Phys. B 107 (2012) 585-589.

[16] J. Egermann, T. Seeger, A. Leipertz, Application of 266-nm and 355$\mathrm{nm}$ Nd:YAG laser radiation for the investigation of fuel-rich sooting hydrocarbon flames by Raman scattering, Appl. Opt. 43 (29) (2004) $5564-5574$.

[17] R. S. Barlow, M. J. Dunn, M. S. Sweeney, S. Hochgreb, Effects of preferential transport in turbulent bluff-body-stabilized lean premixed CH4/air flames, Combust. Flame 159 (8) (2012) 2563 - 2575.

[18] F. Fuest, R. S. Barlow, D. Geyer, F. Seffrin, A. Dreizler, A hybrid method for data evaluation in 1-D Raman spectroscopy, Proc. Combust. Inst. 33 (1) (2011) $815-822$. 
[19] M. S. Sweeney, S. Hochgreb, M. J. Dunn, R. S. Barlow, The structure of turbulent stratified and premixed methane/air flames I: Non-swirling flows, Combust. Flame 159 (9) (2012) 2896 - 2911.

[20] J. Kojima, Q.-V. Nguyen, Laser pulse-stretching with multiple optical ring cavities, Appl. Opt. 41 (30) (2002) 6360-6370.

[21] R. S. Barlow, G. H. Wang, P. Anselmo-Filho, M. S. Sweeney, S. Hochgreb, Application of Raman/Rayleigh/LIF diagnostics in turbulent stratified flames, Proc. Combust. Inst. 32 (1) (2009) 945 - 953.

[22] I. W. Ekoto, R. S. Barlow, Development of a Raman spectroscopy technique to detect alternate transportation fuel hydrocarbon intermediates in complex combustion environments, Sandia national Laboratories Report SAND2012-10389 URL http://prod.sandia.gov/ techlib/access-control.cgi/2012/1210389.pdf.

[23] G. Herzberg, Molecular spectra and molecular structure Vol. II, Infrared and Raman spectra of polyatomic molecules, Krieger Publishing Company, Malabar, Florida, 1991.

[24] D. A. Stephenson, Raman cross sections of selected hydrocarbons and freons, J. Quant. Spectrosc. Radiat. Transfer 14 (12) (1974) 1291 - 1301.

[25] W. Wiegeler, P. Bleckmann, Calculation of relative Raman intensities: II. Calculations using an extended Hückel valence basis set, J. Mol. Struct. 66 (1980) $273-280$. 
[26] W. Meier, O. Keck, Laser Raman scattering in fuel-rich flames: background levels at different excitation wavelengths, Meas. Sci. Technol. 13 (2002) 741-749.

[27] D. A. Long, The Raman Effect: A Unified Treatment of the Theory of Raman Scattering by Molecules, Wiley, 2002.

[28] A. Eckbreth, Laser Diagnostics for Combustion Temperature and Species, Energy and Engineering Science, Taylor \& Francis, 1998.

[29] CHEMKIN-PRO 15131, Reaction Design: San Diego, 2013.

[30] Z. Zhao, M. Chaos, A. Kazakov, F. L. Dryer, Thermal Decomposition Reaction and a Comprehensive Kinetic Model of Dimethyl Ether, Int. J. Chem. Kinet. 40 (2008) 1-18.

[31] K. Seshadri, F. A. Williams, Laminar flow between two plates with injection of a reactant at high Reynolds number, Int. J. Heat Mass Transfer 21 (1978) 251-253.

[32] J. Wang, M. Chaos, B. Yang, T. A. Cool, F. L. Dryer, T. Kasper, N. Hansen, P. Owald, K. Kohse-Höinghaus, P. R. Westmoreland, Composition of reaction intermediates for stoichiometric and fuel-rich dimethyl ether flames: flame-sampling mass spectrometry and modeling studies, Physical Chemistry Chemical Physics 11 (9) (2009) 1265 1460.

[33] B. A. V. Bennett, C. S. McEnally, L. D. Pfefferle, M. D. Smooke, M. B. Colket, Computational and experimental study of the effects of adding 
dimethyl ether and ethanol to nonpremixed ethylene/air flames, Comb. Flame 156 (2009) 1289 - 1302. 


\section{List of Figures}

1 Schematic of the silicon carbide heater. Note that a fiberglass insulation that surrounded the quartz tube is not shown. . . . 29

2 Perpendicular polarized Raman spectrum in a laminar DME/air jet flame at $1290 \mathrm{~K}$ (28\% DME). One hundred laser pulses were averaged. The vertical lines mark the binning regions for the channels of particular molecules. A new region to capture the Raman signal from acetylene is introduced around $1950 \mathrm{~cm}^{-1}$. Approximate volume percentages for each species are given at each channel. An averaged darkfield image and constant background, determined from the 'bgr' channel around $4400 \mathrm{~cm}^{-1}$ were subtracted from the raw data. A channel 'F560' around $900 \mathrm{~cm}^{-1}$ is used for interference correction in the applied matrix inversion method. . . . . . . . . . . . . . . 30

3 Raman responses for $\mathrm{N}_{2}$ and $\mathrm{CO}$ using detailed calculations as outlined in [18] compared to simple harmonic oscillator approximations according to Eq. (3). . . . . . . . . . . . . . . . 31

4 Measured and assumed temperature dependence of the Raman signal from acetylene, referenced to signals at $290 \mathrm{~K}$. Measurements from $290 \mathrm{~K}$ to $700 \mathrm{~K}$ are from electrically heated gas as discussed in Sec. 2. Results from the harmonic oscillator model according to Eq. (3) are shown along with a high-temperature polynomial extrapolation (solid line) similar to that previously used for $\mathrm{N}_{2}$ and $\mathrm{CO}$ [18]. Two additional polynomial extrapolations, corresponding to extreme bounds of the temperature dependence, are used for sensitivity analysis. . . . . . . . . . 31

5 Temperature dependence (relative to $290 \mathrm{~K}$ ) of the $\mathrm{C}_{2}$ and broadband interference on the $\mathrm{C}_{2} \mathrm{H}_{2}$ channel (black line). Also shown is the temperature dependence of the residual background on the $\mathrm{C}_{2} \mathrm{H}_{2}$ channel (red line) most likely corresponding to flame luminosity. . . . . . . . . . . . . . . . . . .

6 (a) Measured acetylene mole fractions and temperature in a laminar DME/air jet flame (20\% DME). (b) Signal-to-noise ratio of acetylene mole fractions with and without application of the interference correction (F560). (c) Interfering signal contributions (\%) of the measured signal on the acetylene channel. These interferences are subtracted during the data-processing. 
$7 \quad$ Measured spatial profiles of the seven major species and temperature from a laminar DME/air flame at $10 \mathrm{~mm}$ downstream of the nozzle. Each point represents the mean from 150 singleshot measurements. Also shown are laminar flame calculations at a strain rate of $28 \mathrm{~s}^{-1}$ using multi-component transport (solid lines). . . . . . . . . . . . . . . . . . . . . 34

8 Conditionally-averaged profiles of the seven major species and temperature (points with lines included to guide eyes) as a function of mixture fraction from a laminar DME/air flame at $10 \mathrm{~mm}$ downstream of the nozzle. Also shown are laminar flame calculations at a strain rate of $28 \mathrm{~s}^{-1}$ using multicomponent transport (solid lines). . . . . . . . . . . . . . 34

$9 \quad$ Measured spatial profiles of acetylene and temperature (points) from a laminar DME/air flame at $10 \mathrm{~mm}$ downstream of the nozzle. Each point represents the mean from 150 single-shot measurements. Also shown are laminar flame calculations at a strain rate of $28 \mathrm{~s}^{-1}$ using multi-component transport (solid lines). . . . . . . . . . . . . . . . . . . 35

10 Measured scatter plots for acetylene and conditionally-averaged profiles of acetylene and temperature (points) from a laminar DME/air flame at $10 \mathrm{~mm}$ downstream of the nozzle. Also shown are laminar flame calculations at a strain rate of $28 \mathrm{~s}^{-1}$ using multi-component transport (solid lines). . . . . . . . . 35

11 Impact of different response curves and crosstalk corrections described in Section 4 on the measured acetylene mole fractions. 36

12 Measured scatter plots of acetylene and conditionally-averaged profiles of acetylene and temperature (points) in a piloted turbulent partially-premixed DME/air flame at an axial position of $\mathrm{x} / \mathrm{d}=5 . \ldots \ldots \ldots \ldots$. . . . . . . . . . . 37 


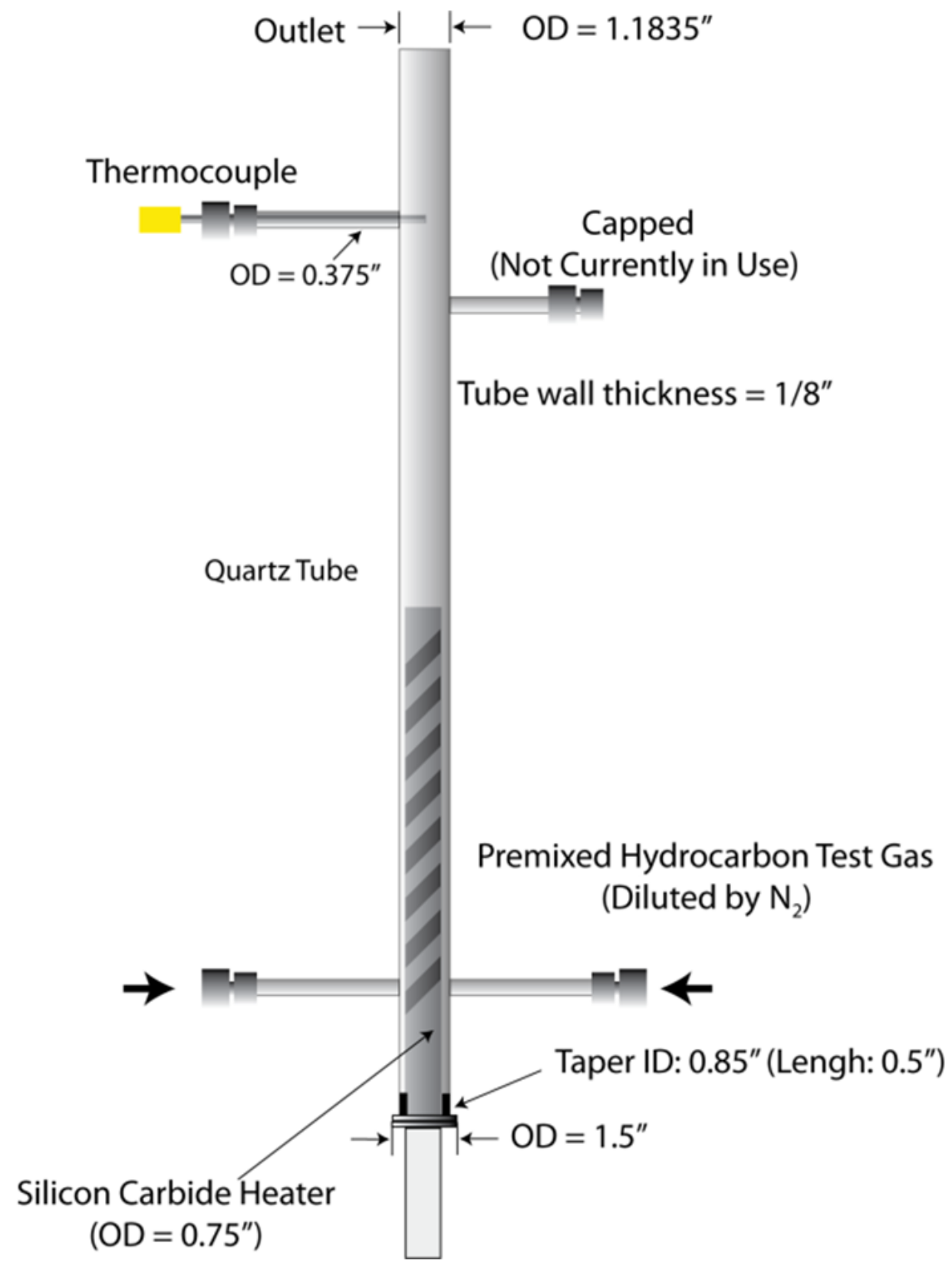

Figure 1: Schematic of the silicon carbide heater. Note that a fiberglass insulation that surrounded the quartz tube is not shown. 


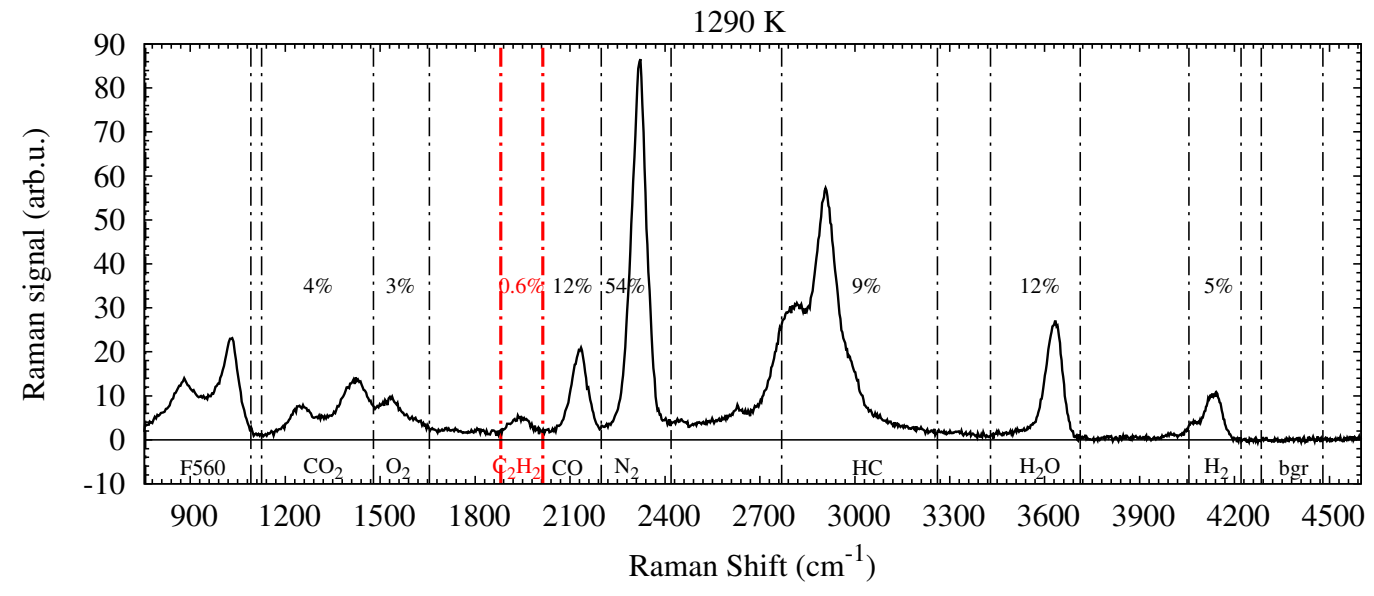

Figure 2: Perpendicular polarized Raman spectrum in a laminar DME/air jet flame at $1290 \mathrm{~K}$ (28\% DME). One hundred laser pulses were averaged. The vertical lines mark the binning regions for the channels of particular molecules. A new region to capture the Raman signal from acetylene is introduced around $1950 \mathrm{~cm}^{-1}$. Approximate volume percentages for each species are given at each channel. An averaged darkfield image and constant background, determined from the 'bgr' channel around $4400 \mathrm{~cm}^{-1}$ were subtracted from the raw data. A channel 'F560' around $900 \mathrm{~cm}^{-1}$ is used for interference correction in the applied matrix inversion method. 


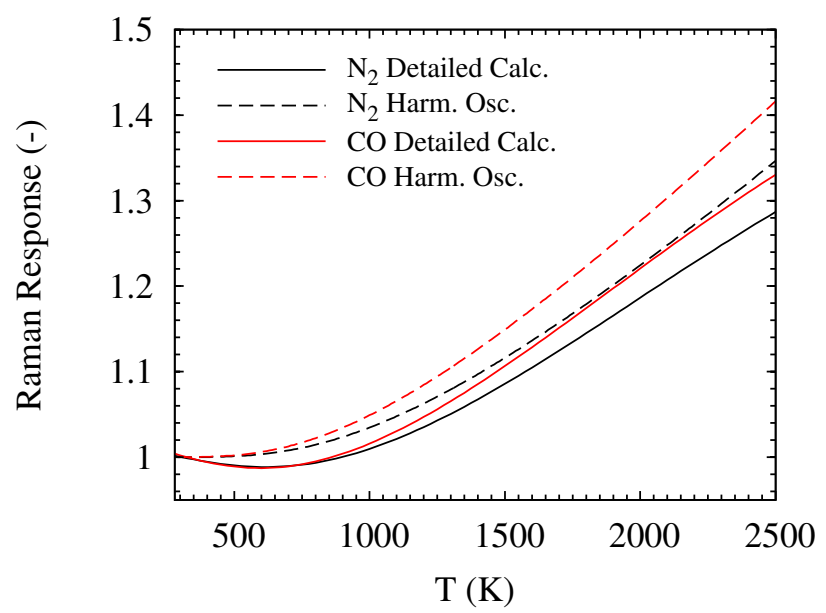

Figure 3: Raman responses for $\mathrm{N}_{2}$ and $\mathrm{CO}$ using detailed calculations as outlined in [18] compared to simple harmonic oscillator approximations according to Eq. (3).

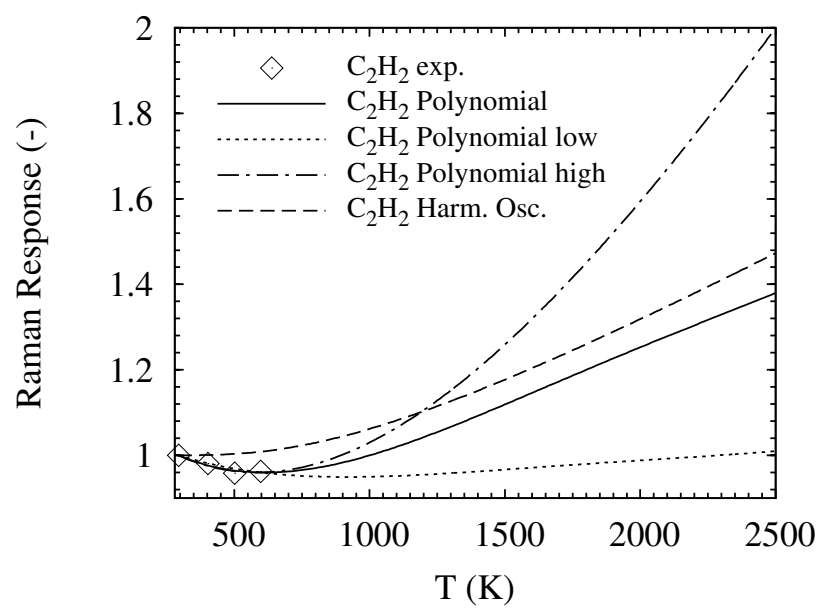

Figure 4: Measured and assumed temperature dependence of the Raman signal from acetylene, referenced to signals at $290 \mathrm{~K}$. Measurements from $290 \mathrm{~K}$ to $700 \mathrm{~K}$ are from electrically heated gas as discussed in Sec. 2. Results from the harmonic oscillator model according to Eq. (3) are shown along with a high-temperature polynomial extrapolation (solid line) similar to that previously used for $\mathrm{N}_{2}$ and $\mathrm{CO}$ [18]. Two additional polynomial extrapolations, corresponding to extreme bounds of the temperature dependence, are used for sensitivity analysis. 


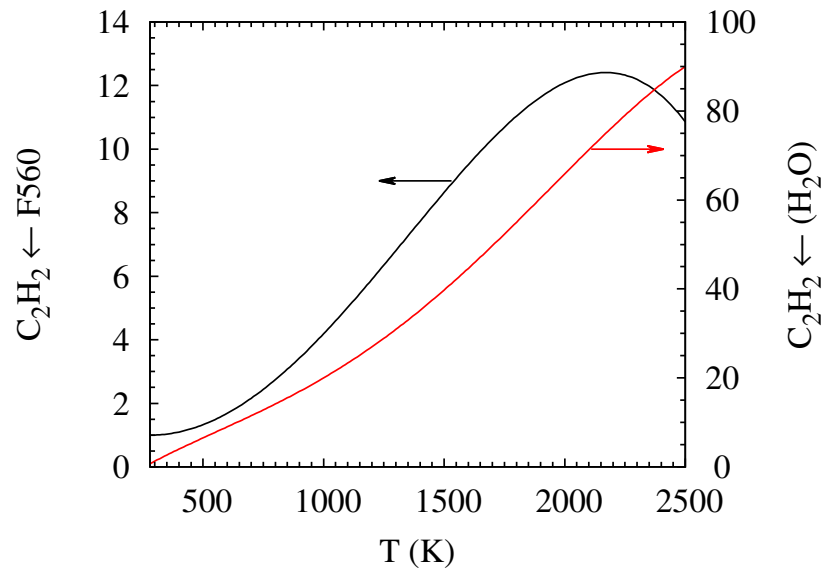

Figure 5: Temperature dependence (relative to $290 \mathrm{~K}$ ) of the $\mathrm{C}_{2}$ and broadband interference on the $\mathrm{C}_{2} \mathrm{H}_{2}$ channel (black line). Also shown is the temperature dependence of the residual background on the $\mathrm{C}_{2} \mathrm{H}_{2}$ channel (red line) most likely corresponding to flame luminosity. 

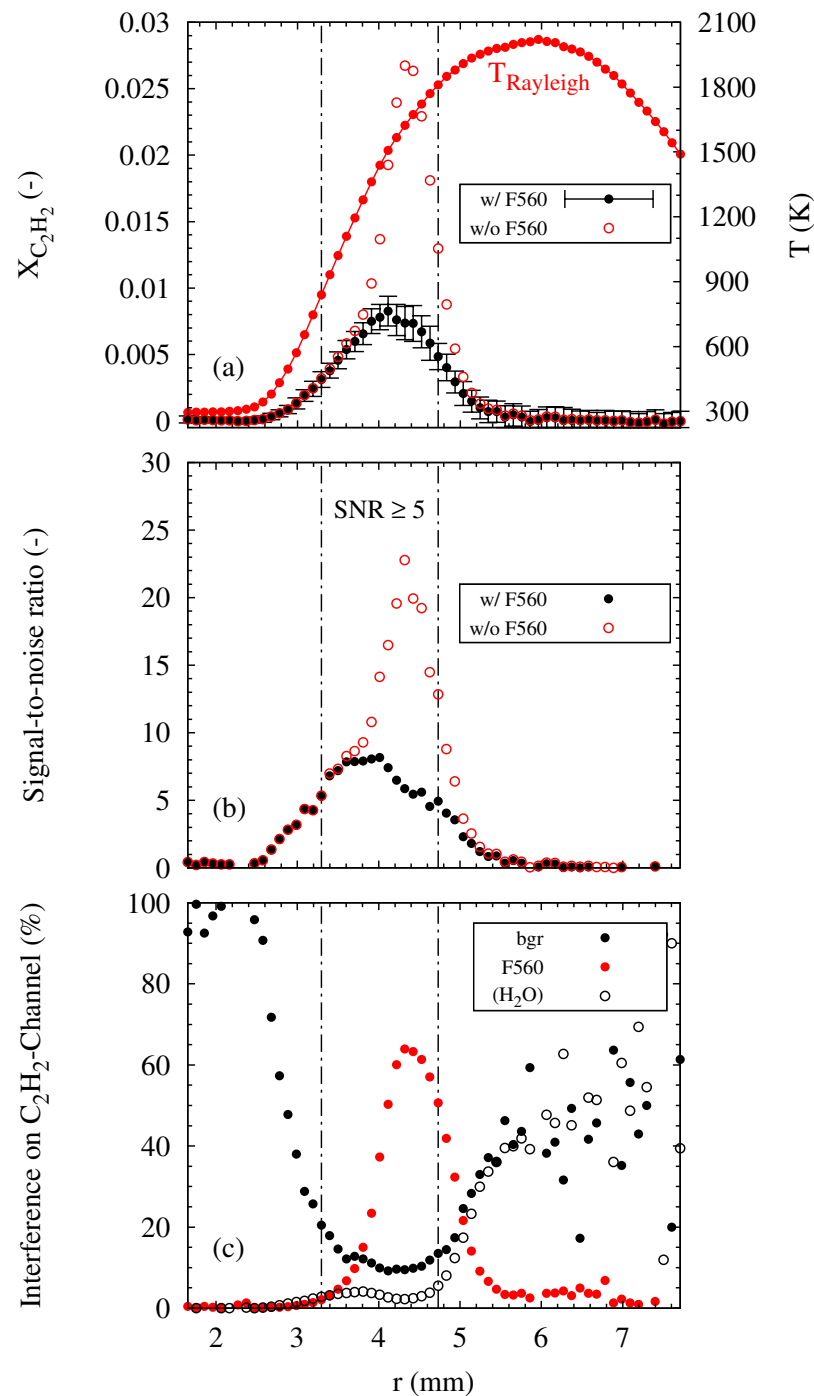

Figure 6: (a) Measured acetylene mole fractions and temperature in a laminar DME/air jet flame (20\% DME). (b) Signal-to-noise ratio of acetylene mole fractions with and without application of the interference correction (F560). (c) Interfering signal contributions (\%) of the measured signal on the acetylene channel. These interferences are subtracted during the data-processing. 


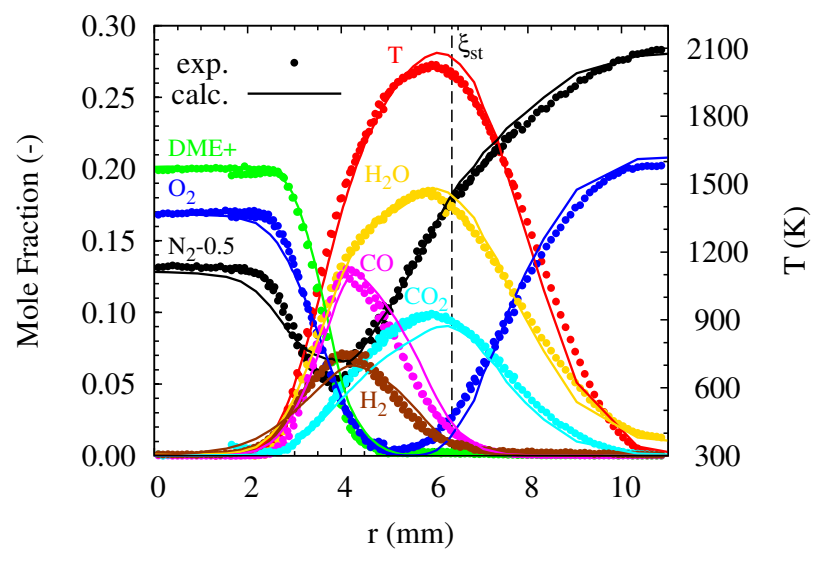

Figure 7: Measured spatial profiles of the seven major species and temperature from a laminar DME/air flame at $10 \mathrm{~mm}$ downstream of the nozzle. Each point represents the mean from 150 single-shot measurements. Also shown are laminar flame calculations at a strain rate of $28 \mathrm{~s}^{-1}$ using multi-component transport (solid lines).

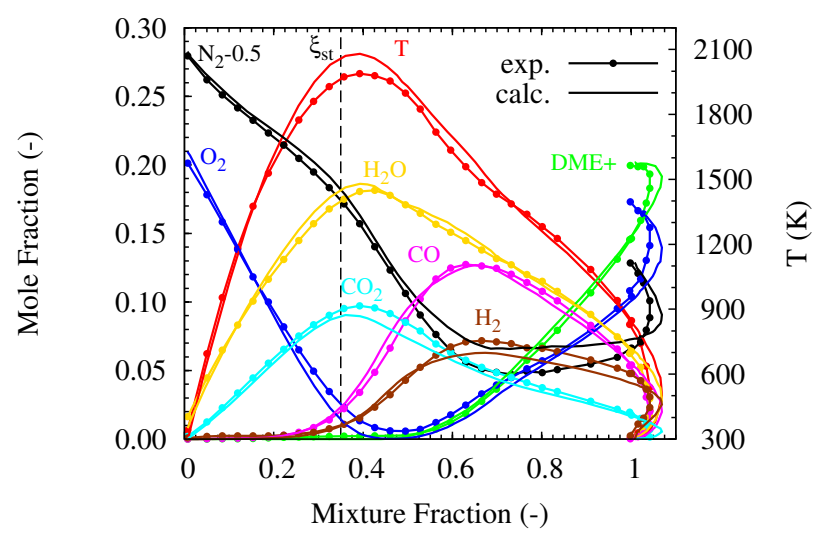

Figure 8: Conditionally-averaged profiles of the seven major species and temperature (points with lines included to guide eyes) as a function of mixture fraction from a laminar DME/air flame at $10 \mathrm{~mm}$ downstream of the nozzle. Also shown are laminar flame calculations at a strain rate of $28 \mathrm{~s}^{-1}$ using multi-component transport (solid lines). 


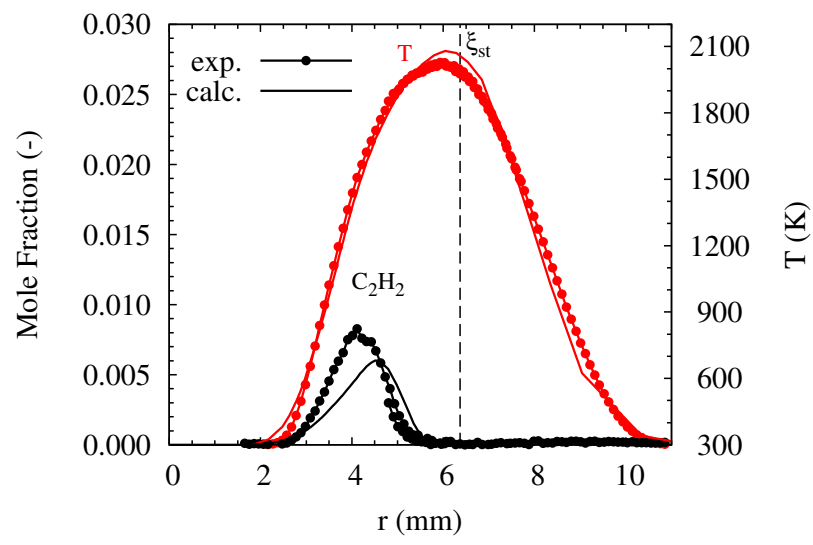

Figure 9: Measured spatial profiles of acetylene and temperature (points) from a laminar $\mathrm{DME} /$ air flame at $10 \mathrm{~mm}$ downstream of the nozzle. Each point represents the mean from 150 single-shot measurements. Also shown are laminar flame calculations at a strain rate of $28 \mathrm{~s}^{-1}$ using multi-component transport (solid lines).

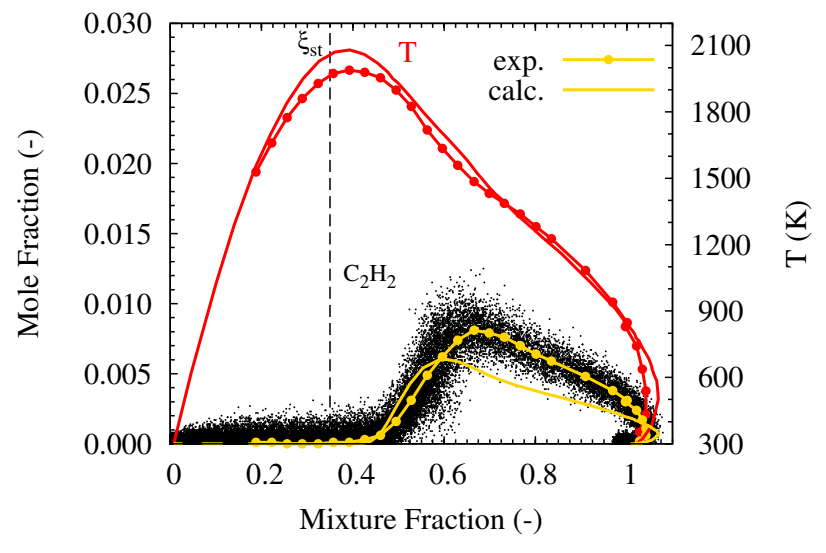

Figure 10: Measured scatter plots for acetylene and conditionally-averaged profiles of acetylene and temperature (points) from a laminar DME/air flame at $10 \mathrm{~mm}$ downstream of the nozzle. Also shown are laminar flame calculations at a strain rate of $28 \mathrm{~s}^{-1}$ using multi-component transport (solid lines). 


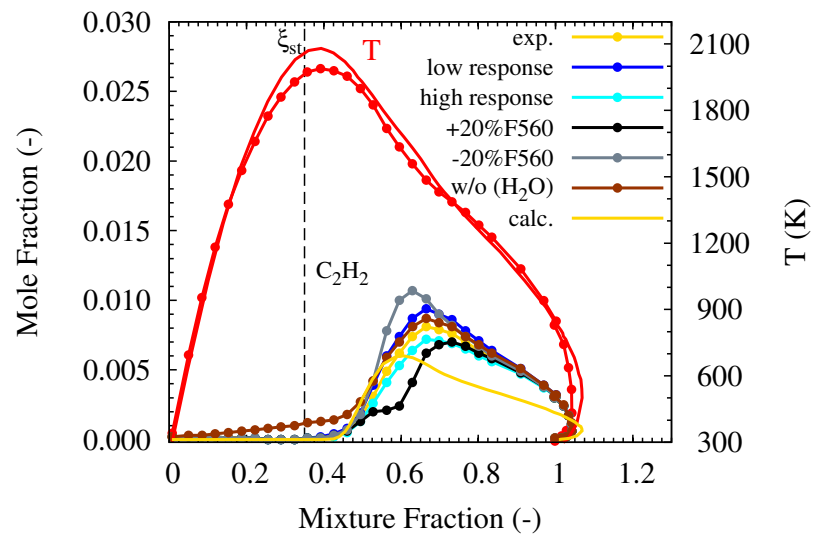

Figure 11: Impact of different response curves and crosstalk corrections described in Section 4 on the measured acetylene mole fractions. 


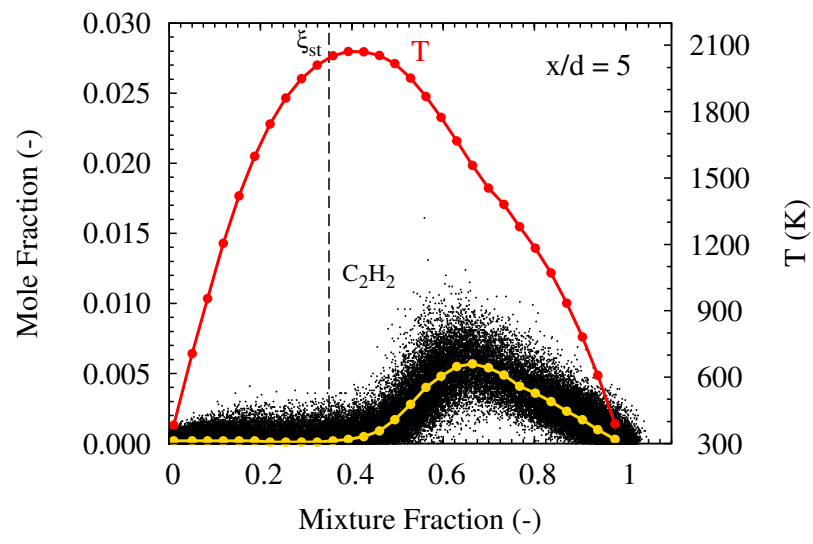

Figure 12: Measured scatter plots of acetylene and conditionally-averaged profiles of acetylene and temperature (points) in a piloted turbulent partially-premixed DME/air flame at an axial position of $\mathrm{x} / \mathrm{d}=5$. 